\title{
Effect of benzalkonium chloride on dentin bond strength and endogenous enzymatic activity
}

\author{
Allegra Comba ${ }^{\mathrm{a}}$, Tatjana Maravic ${ }^{\mathrm{a}}$, Lucrezia Valente ${ }^{\mathrm{a}}$, Margherita Girlando ${ }^{\mathrm{a}}$, Sandra R Cunha ${ }^{\mathrm{b}}$, \\ Vittorio Checchi ${ }^{\mathrm{a}}$, Stefano Salgarello ${ }^{\mathrm{c}}$, Franklin R Tay ${ }^{\mathrm{d}}$, Nicola Scotti ${ }^{\mathrm{e}}$, Lorenzo Breschi ${ }^{\mathrm{a}, *}$, \\ Annalisa Mazzoni ${ }^{\mathrm{a}}$ \\ a Department of Biomedical and Neuromotor Sciences, DIBINEM, University of Bologna - Alma Mater Studiorum, Via San Vitale 59, 40125 Bologna, Italy \\ ${ }^{\mathrm{b}}$ Department of Restorative Dentistry, School of Dentistry, University of São Paulo, Av. Prof. Lineu Prestes 2227, 05508-000 São Paulo, Brazil \\ ${ }^{\mathrm{c}}$ Dipartimento di Specialità Medico-Chirurgiche, Scienze Radiologiche e Sanità Pubblica - Università degli Studi di Brescia, P.le Spedali Civili 1, 25123 Brescia, Italy \\ ${ }^{\mathrm{d}}$ The Dental College of Georgia, Augusta University, 1430 John Wesley Gilbert Drive, Augusta, GA, USA \\ ${ }^{\mathrm{e}}$ Department of Surgical Sciences, University of Turin, Dental School Lingotto, Via Nizza 230, 10100 Torino, Italy
}

\section{A R T I C L E I N F O}

\section{Keywords:}

Benzalkonium chloride

Universal adhesives

MMPs

In situ zymography

Zymographic assay

Microtensile bond strength

\begin{abstract}
A B S T R A C T
Objective: This in vitro study evaluated at baseline (T0) and over time (T12 months), the effect of a multi-mode universal adhesive compared with two experimental formulations blended with different concentrations of benzalkonium chloride (BAC), on bond strength and endogenous enzymatic activity.

Methods and materials: Specimens were assigned to the following groups according to the adhesive protocol: G1) All-Bond Universal (ABU) self-etch (SE); G2) ABU + 0.5\% BAC SE; G3) ABU + 1\% methacrylate BAC SE; G4) $\mathrm{ABU}$ etch-and-rinse (E\&R); G5) $\mathrm{ABU}+0.5 \%$ BAC E\&R; G6) $\mathrm{ABU}+1 \%$ methacrylate BAC E\&R. Gelatin zymography was performed on dentin powder obtained from eight human third molars. Endogenous enzymatic activity within the hybrid layer was examined using in situ zymography after $24 \mathrm{~h}$ (T0) or 1-year storage in artificial saliva (T12). Forty intact molars were prepared for microtensile bond strength test at T0 and T12. Results were statistically analyzed with three-way ANOVA $(\alpha=0.05)$.

Results: Gelatin zymography assay and in situ zymography quantification analyses indicated that all the BACcontaining formulations decreased matrix metalloproteinase expression. However, in situ zymography showed a general trend of enzymatic activity increase after aging. Microtensile bond-strength testing showed decrease in bond strength over time in all the tested groups; performances of the $1 \%$ methacrylate BAC experimental groups were worse than the control.

Conclusions: BAC-containing adhesives reduce endogenous enzymatic activity both immediately and over time. However, independently from the adhesive employed, increase in the gelatinolytic activity over time and decrease in bond strength was found (especially in the BAC $+1 \%$ methacrylate groups), probably due to impaired polymerization properties.

Clinical significance: Adhesives containing protease inhibitors are practical and efficient tools in clinical practice for enhancement of the longevity of dental restorations. However, extensive investigation of the mechanical and adhesive properties of the material is necessary prior to their clinical use.
\end{abstract}

\section{Introduction}

Adhesive systems introduced a revolution in contemporary restorative dentistry by enabling previously inconceivable treatment plans. Currently available adhesive systems allow clinicians to bond to tooth structure without the need for a retentive cavity design because they provide immediate bond strength to the dental substrate [1].

Regardless of advancements in adhesive dentistry over the past decade, the stability of resin-dentin bonds over time remains an issue that has not been adequately addressed [1]. Adhesion to dentin is a type

\footnotetext{
* Corresponding author.

E-mail addresses: allegra.comba@unibo.it (A. Comba), tatjana.maravic2@unibo.it (T. Maravic), lucrezia.valente@studio.unibo.it (L. Valente), margherita.girlando53@gmail.com (M. Girlando), sandra.cunha@gmail.com (S.R. Cunha), vchecchi@hotmail.com (V. Checchi), stefano.salgarello@unibs.it (S. Salgarello), tayfranklin7@gmail.com (F.R. Tay), nscotti@unito.it (N. Scotti), lorenzo.breschi@unibo.it (L. Breschi), annalisa.mazzoni@unibo.it (A. Mazzoni).
} 
of in situ tissue engineering that creates a hybrid layer (HL) in dentin by infiltration of the demineralized collagen fibril network with adhesive resin monomers [2-4]. Although this interdiffusion layer is responsible for the retention of resin restorations, it is the most vulnerable component of the resin-dentin bond [5]. In vitro and in vivo studies showed that the degradation of resin-dentin bonds over time is caused by the hydrolytic breakdown of the resin and denuded dentinal collagen fibrils at the base of the HL $[6,7]$. Host-derived proteinases are responsible for the deterioration of the HL with aging [8-11].

Among the endogenous enzymes, matrix metalloproteinases (MMPs) and cysteine cathepsins are involved in the hydrolytic degradation of dentin collagen, with a 36-70\% decrease in bond strength after 1 year of storage in artificial saliva [7,12]. Accordingly, increasing the resistance of collagen fibrils against enzymatic deterioration and inactivating of the activities of proteinases are fundamental approaches to improve the longevity of resin-dentin bonds $[11,13]$.

Several natural and synthetic MMPs inhibitors (chlorhexidine, galardin, quaternary ammonium methacrylates, various collagen crosslinking agents) have been employed experimentally as part of the bonding procedures on dentin with the intention of enhancing HL longevity [10,14-20]. Benzalkonium chloride (BAC), an antimicrobial agent containing a quaternary ammonium group, has demonstrated inhibitory potential against endogenous proteases [18]. A commercially available antimicrobial phosphoric acid etchant containing $1.0 \mathrm{wt} \%$ BAC (Etch-37 w/BAC, Bisco Inc, Schaumburg, IL, USA) is commercially available and does not immediate bond strength [21]. The etchant possesses anti-proteolytic properties. However, rinsing of the BACcontaining etchant in the etch-and-rinse bonding technique limits the amount that of BAC available in the hybrid layer and hence, its antiproteolytic potential [22]. A more effective delivery system may entail incorporation of BAC into the primer and/or adhesive formulation to obtain deeper infiltration of the agent into dentin without lengthening the clinical procedures.

The objective of the present in vitro study was to evaluate at baseline (T0) and over time (T12 months), the effect of a commercially available multi-mode universal adhesive compared with two experimental formulations of this adhesive blended with different concentrations of BAC $(0.5 \%$ BAC or $1 \%$ methacrylate BAC) on endogenous enzymatic activity and bond strength. The null hypotheses tested were that incorporation of BAC in an adhesive formulation: 1) has no effect on the activity of endogenous dentin MMPs immediately or over time; 2) has no effect on immediate bond strength and bond strength after aging for 12 months.

\section{Materials and methods}

\subsection{Gelatin zymography of dentin powder extracts}

Zymography was performed in accordance with a previous publication [23]. Briefly, mineralized dentin powder was obtained from eight human third molars by triturating dentin frozen in liquid nitrogen using a Retsch mill (MM400, Retsch GmbH, Haan, Germany). Aliquots of mineralized dentin powder $(100 \mathrm{mg})$ were treated as follows:

- L1: dentin remained mineralized (DP, control);

- L2: demineralized with $10 \mathrm{wt} \%$ phosphoric acid for $10 \mathrm{~min}$ at $4{ }^{\circ} \mathrm{C}$ to simulate the first step of the etch-and-rinse (E\&R) approach (DDP);

- L3: All-Bond Universal adhesive (ABU) applied on DP for $30 \mathrm{~min}$ to simulate the self-etch (SE) approach;

- L4: ABU + 0.5\% BAC applied on DP for $30 \mathrm{~min}$ to simulate the SE approach;

- L5: $\mathrm{ABU}+1 \%$ methacrylate BAC applied on DP for $30 \mathrm{~min}$ to simulate the SE approach;

- L6: DDP (as for L2) treated with ABU for 30 min to simulate the E\&R approach;

- L7: DDP treated with $\mathrm{ABU}+0.5 \% \mathrm{BAC}$ for 30 min to simulate the $\mathrm{E}$ \&R approach;
- L8: DDP treated with $\mathrm{ABU}+1 \%$ methacrylate BAC for $30 \mathrm{~min}$ to simulate the E\&R approach.

All treatments involving adhesive resins were performed with light protection at $4{ }^{\circ} \mathrm{C}$ under constant agitation. After treatment, the specimens were rinsed several times with acetone and centrifuged at $4{ }^{\circ} \mathrm{C}$ $(20,800 \mathrm{G})$ to remove the adhesive resins. After the aforementioned treatments, the dentin powder aliquots were suspended in extraction buffer ( $50 \mathrm{mM}$ Tris- $\mathrm{HCl} \mathrm{pH} 6$, containing $5 \mathrm{mM} \mathrm{CaCl}_{2}, 100 \mathrm{mM} \mathrm{NaCl}$, $0.1 \%$ Triton X-100, $0.1 \%$ nonionic detergent P- $40,0.1 \mathrm{mM} \mathrm{ZnCl}_{2}$, $0.02 \% \mathrm{NaN}_{3}$ ) for $24 \mathrm{~h}$ at $4{ }^{\circ} \mathrm{C}$. They were further sonicated for $10 \mathrm{~min}$ (ca. $\approx 30$ pulses) and centrifuged two times for $20 \mathrm{~min}$ at $4{ }^{\circ} \mathrm{C}$ $(20,800 \mathrm{G})$ to collect the supernatant and remove powder remnants. The supernatant was concentrated using Vivaspin concentrators (10,000 kDa cut off; Vivaspin Sartorius Stedim Biotech, Goettingen, Germany) in several rounds of centrifugation of $20 \mathrm{~min}$ each at $25^{\circ} \mathrm{C}$ $(15,000 \mathrm{G})$. Bradford assay was used to determine the total protein concentration in the dentin protein extracts. The dentin protein aliquots were diluted in Laemmli sample buffer in a 4:1 ratio and subjected to electrophoresis under non-reducing conditions in $10 \%$ sodium dodecyl sulfate-polyacrylamide gel electrophoresis (SDS-PAGE) containing $1 \mathrm{mg} / \mathrm{m}$ L fluorescein-labeled gelatin. Pre-stained low-range molecular weight SDS-PAGE standards (Bio-Rad, Hercules, CA) were used as molecular-weight markers. After electrophoresis, the gels were washed for $1 \mathrm{~h}$ in $2 \%$ Triton X-100 and incubated in zymography activation buffer $\left(50 \mathrm{mmol} / \mathrm{L}\right.$ Tris- $\mathrm{HCl}, 5 \mathrm{mmol} / \mathrm{L} \mathrm{CaCl}_{2}, \mathrm{pH}$ 7.4) for $48 \mathrm{~h}$. Proteolytic activity was evaluated using a long-wave ultraviolet light scanner (Chemi-Doc Universal Hood, Bio-Rad, Hercules, CA, USA). Gelatinase activities in the samples were analyzed in duplicate by gelatin zymography. Densitometric evaluation of bands obtained from zymography was performed using the ImageJ software (National Institutes of Health, Bethesda, MD, USA).

\subsection{In-situ zymography of resin-dentin interfaces}

One millimeter-thick slabs of middle/deep dentin were obtained from three extracted human third molars using a low-speed saw (Micromet, Remet; Bologna, Italy) with water-cooling. Two slabs were obtained from each tooth and then further divided into 4 pieces, so that testing of all groups was performed using the same dentinal substrate. A standardized smear layer was created on each dentin surface using 600grit silicon carbide paper. The dentin specimens were further treated as follows:

- Group 1 (G1): ABU was directly applied on untreated dentin according to the manufacturer's instructions for the SE mode.

- Group 2 (G2): ABU + 0.5\% BAC was directly applied on untreated dentin in the same manner as $\mathrm{ABU}$.

- Group 3 (G3): ABU + 1\% methacrylate BAC was directly applied on untreated dentin in the same manner as $\mathrm{ABU}$ for the SE mode.

- Group 4 (G4): dentin surfaces were etched with 35\% phosphoricacid gel ( $3 \mathrm{M}$ ESPE, St. Paul, MN, USA) for $15 \mathrm{~s}$ and rinsed with water. $\mathrm{ABU}$ was generously applied according to the manufacturer's instructions in the E\&R mode.

- Group 5 (G5): dentin surfaces were etched as in G4. ABU + 0.5\% BAC was generously applied in the same manner as ABU.

- Group 6 (G6): dentin surfaces were etched as in G4. ABU + 1\% methacrylate BAC was generously applied in the same manner as ABU.

Each bonded specimen was light-cured for $20 \mathrm{~s}$ using a LED lightcuring unit (Demi Plus, Kerr Corp., Middleton, USA). The adhesivedentin interfaces were then exposed by cutting the bonded specimens vertically into 1 -mm-thick sticks using a slow-speed saw with water cooling. The sticks were glued to glass slides, ground down and polished to obtain specimens of approximately $50 \mu \mathrm{m}$ thick, using a series 


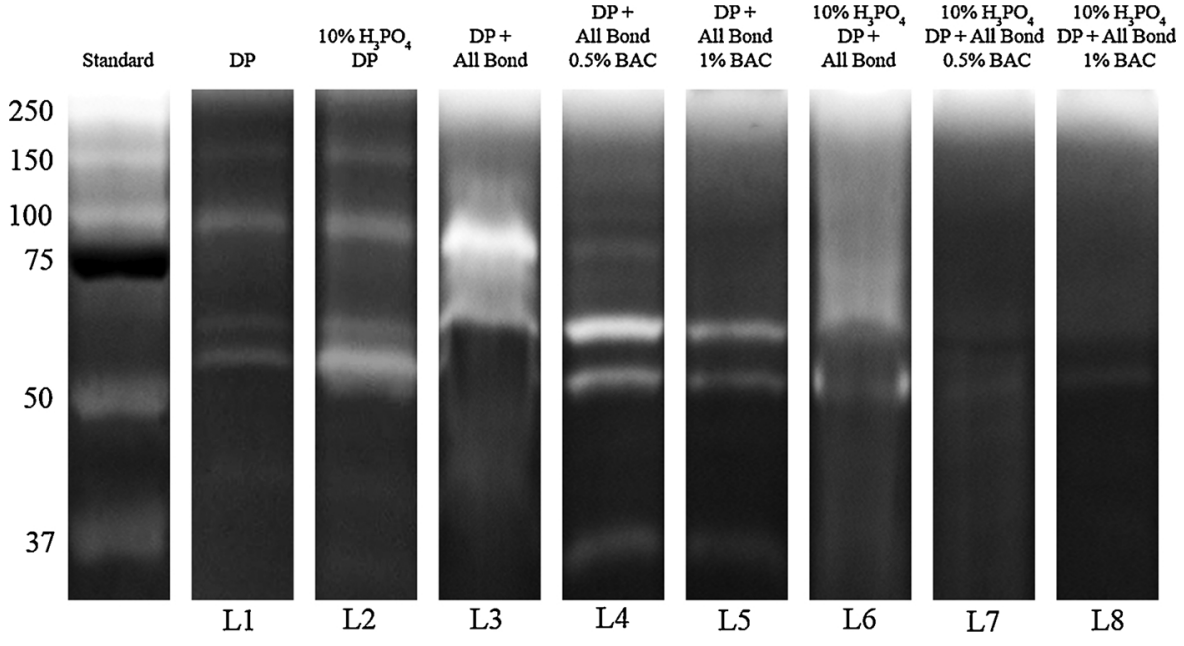

Fig. 1. Zymographic analysis of proteins extracted from dentin powder. Std: Standards (Std); L1: mineralized dentin (DP) showing the presence of activity of pro-form of MMP-9 (92 kDa) and active form of MMP2 (66 kDa); L2: demineralized dentin powder (DDP) showing an increase of pro-form of MMP-9 (92 kDa), active form of MMP-2 (66 kDa); L3: DP after incubation with All-Bond Universal (ABU) showing activity of active form MMP-9 (86 kDa) and MMP-2 (66 kDa); L4: DP after incubation with ABU $+0.5 \%$ BAC showing a reduction in the activity of MMP-9 active form and an increase in MMP-2 activity; L5: DP after incubation with $\mathrm{ABU}+1 \%$ methacrylate $\mathrm{BAC}$ showing inhibition of MMP-9 and a decrease in the activity of MMP-2 ; L6: DDP after incubation with ABU showing MMP-2 activity and inhibition of MMP-9 ; L7: DDP after incubation with $\mathrm{ABU}+0.5 \%$ BAC showing inhibition of MMP-9 and a reduction in MMP-2 activity; L8: DDP treated with $\mathrm{ABU}+1 \%$ methacrylate $\mathrm{BAC}$ showing an enzymatic activity similar to that of L7. of wet silicon carbide papers. Self-quenched fluorescein-conjugated gelatin was used as the MMP substrate (E-12055, Molecular Probes, Eugene, OR, USA) for in situ zymography at the baseline (T0) and after 12 months (T12) of storage in artificial saliva at $37^{\circ} \mathrm{C}$ as previously described [24]. Briefly, the fluorescent gelatin mixture was placed on top of each slab and covered with a coverslip. The slides were incubated in a humidified chamber at $37^{\circ} \mathrm{C}$ overnight. During incubation, the assemblies were prevented from direct contact with water and were protected from exposure to light. After incubation, the microscopic slides were examined using a confocal laser scanning microscope (excitation wavelength $488 \mathrm{~nm}$; emission wavelength $530 \mathrm{~nm}$; Model A1-R; Nikon, Tokyo, Japan). For each specimen, a series of images were made to visualize hydrolysis of the quenched fluorescein-conjugated gelatin substrate as an indicator of endogenous gelatinolytic activity. Enzymatic activity was quantified as the integrated density of the fluorescence signals using ImageJ software. Because the data were not normally distributed even after nonlinear transformation, they were analyzed using the Mann-Whitney U-test ( $\alpha=0.05)$.

\subsection{Microtensile bond strength ( $\mu \mathrm{TBS})$}

Ninety freshly extracted non-carious sound human third molars were collected after the patients' informed consents were obtained under a protocol approved by the institutional review board of the University of Bologna, Italy. Tooth crowns were sectioned with the Micromet diamond saw with water irrigation to expose middle/deep coronal dentin. A standardized smear layer was created on the dentin with wet 600 -grit silicon carbide paper. The teeth were randomly assigned to the same six groups $(\mathrm{N}=15)$ described for in situ zymography and identical bonding procedures were performed. Resin composite buildups (4-mm thick) were produced with 2 incremental layers of Filtek Z-250 (3 M ESPE) on each bonded specimen.

The specimens were sectioned to produce approximately $1-\mathrm{mm}$ thick beams for testing with the microtensile non-trimming technique. The dimension of each beam (ca. $0.9 \mathrm{~mm} \times 0.9 \mathrm{~mm} \times 8 \mathrm{~mm}$ ) was recorded using a pair of digital calipers to the nearest $0.01 \mathrm{~mm}$. The bonded area was calculated for conversion of microtensile strength values (N) to units of stress (MPa). Beams were tested after $24 \mathrm{~h}$ (T0) and storage in artificial saliva at $37{ }^{\circ} \mathrm{C}$ for 12 months (T12). The artificial saliva consisted of $\mathrm{CaCl}_{2}(0.7 \mathrm{mmoles} / \mathrm{L}), \mathrm{MgCl}_{2} 6 \mathrm{H}_{2} \mathrm{O}$ (0.2 mmoles/L), $\mathrm{KH}_{2} \mathrm{PO}_{4}$ (4.0 mmoles/L), $\mathrm{KCl}$ (30 mmoles/L), $\mathrm{NaN}_{3}$ (0.3 mmoles/L) in HEPES buffer [29]. After storage, a simplified universal testing machine (Bisco Inc.) was used to stress the bonded beams to failure at a crosshead speed of $1 \mathrm{~mm} / \mathrm{min}$. Each specimen was observed under a stereomicroscope (Stemi 2000-C; Carl Zeiss Jena GmbH, Göttingen, Germany) at $50 \times$ magnification to determine the failure mode. Failure modes were classified as adhesive (A), cohesive in resin composite (CC) or cohesive in dentin (CD). Premature failures that occurred during sectioning were not included in the statistical analysis because there were less than $3 \%$ debonded beams and they were similarly distributed among the groups.

Analysis was performed using the tooth as the statistical unit. Bond strength data from each tooth were averaged to obtain the mean bond strength for that tooth. The acquired data were evaluated for compliance with the normality (Shapiro-Wilk test) and equality of variance (modified Levine test) assumptions required for parametric statistical analysis. Because those assumptions were not violated, the data were analyzed with a three-factor analysis of variance, to examine the effects of "adhesive system", "aging time" and "bonding strategy" on $\mu$ TBS. Post-hoc pairwise comparisons were conducted using the Tukey method. For all analyses, statistical significance was pre-set at $\alpha=0.05$.

\section{Results}

\subsection{Gelatin zymography}

Zymography results are shown in Figs. 1 and 2. Zymographic assay revealed low expression of pro-form of MMP-9 (92 kDa) and active form of MMP-2 $(66 \mathrm{kDa})$ in the mineralized dentin powder. In contrast, protease expressions in the demineralized powder were more pronounced. When employed in the SE mode, ABU increased the activity of MMP-9 pro-form and MMP-2 active form, while BAC-containing adhesive formulations showed reduced expression of MMP-2, and almost complete inhibition of the bands corresponding to MMP-9. All-Bond Universal adhesive applied in the E\&R mode increased the activity of MMP-2 and inhibited MMP-9, while all the BAC-containing formulations tested in the E\&R mode decreased MMP-2 expression and completely inhibited MMP-9.

\subsection{In situ zymography}

Qualitative and quantitative in situ zymography results are shown in Figs. 3, 4, 5, 6, 7 . There was a decrease in enzymatic activity at T0 in all the experimental groups compared to controls. All the groups showed a general trend of increase in enzymatic activity after aging, except for the ABU SE $+1 \%$ methacrylate BAC group, which showed further decrease. Overall, the experimental groups bonded with $A B U+1 \%$ methacrylate BAC showed the lowest level of fluorescence over time, regardless of the bonding strategy employed $(\mathrm{p}<0.05)$. 


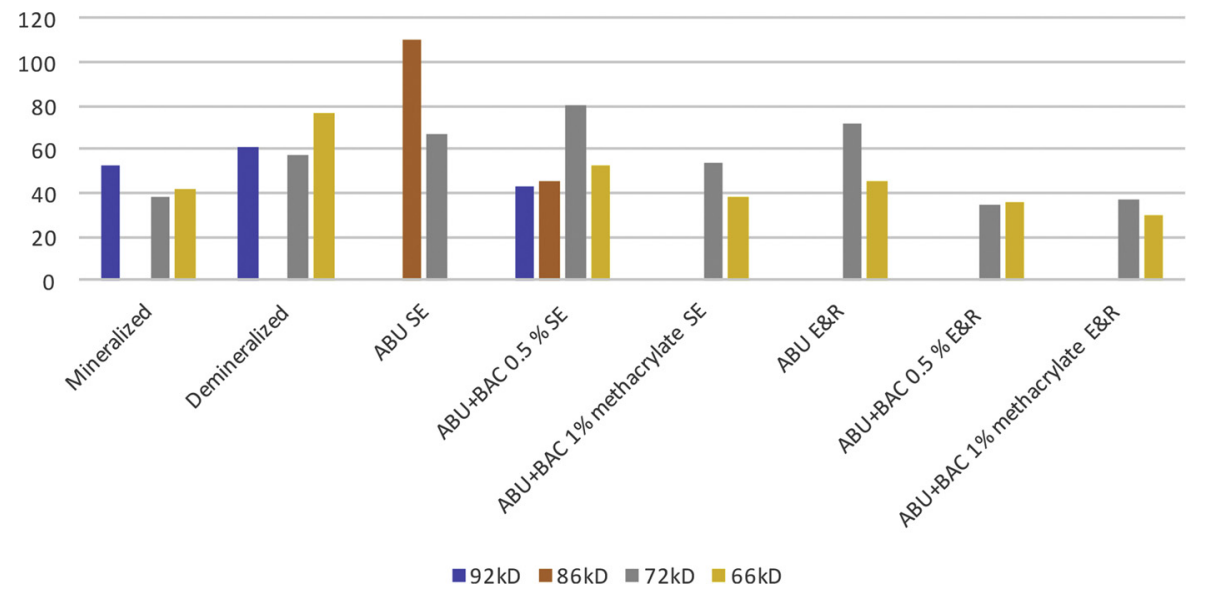

Fig. 2. Zymographic assay quantification showing a generally lower enzymatic activity in BAC-containing groups.

\subsection{Microtensile bond strength}

Bond strength values (means and standard deviations, in MPa) at T0 and T12 months are reported in Table 1. Statistical analysis showed that only the variables "adhesive system" and "aging time" significantly affected the results, but not the variable "bonding strategy". Post-hoc analysis indicated that $\mathrm{ABU}+1 \%$ methacrylate $\mathrm{BAC}$ performed significantly worse than $\mathrm{ABU}$ or $\mathrm{ABU}+0.5 \% \mathrm{BAC}(\mathrm{p}<0.05)$. In adidtion, all groups showed a significant decrease in microtensile bond strength values over time ( $\mathrm{p}<0.05)$.

\section{Discussion}

The present study investigated BAC blended within a commercially available multi-mode adhesive in terms of anti-enzymatic activity and bond strength preservation. The use of two adhesive formulations containing BAC decreased the activity of MMPs both in the dentin protein extracts and in the HL. This warrants rejection of the first null hypothesis that "incorporation of BAC in an adhesive formulation has no effect on the activity of endogenous dentin MMPs immediately or over time". Furthermore, the two formulations of BAC-containing adhesives influenced bond strength differently, both immediately and after aging. Even though aging in artificial saliva significantly reduced adhesion values, irrespective of whether BAC was incorporated in the adhesive formulation or the application mode (ER or SE), 1\% BAC methacrylate formulation showed reduced bond strength values both immediately and over time. Hence, the second null hypothesis that "incorporation of BAC in an adhesive formulation has no effect on immediate bond strength and bond strength after aging for 12 months" has to be rejected.

Regardless of the bonding system or bonding strategy, adhesive resin monomers do not completely coat the completely or partially demineralized collagen fibrils, producing HLs that are susceptible to enzymatic hydrolytic degradation over time [25]. In addition to the enzymatic degradation of the collagen, resin-based restorations are subjected to polymer degradation by enzymes present in the saliva that results in exposure of more collagen. The unprotected collagen fibrils at the base of the hybrid layer are attacked by endogenous enzymes that are bound to the fibrils, causing the loss of the anchoring function of the HL and bond strength degradation over time [26]. These events occur
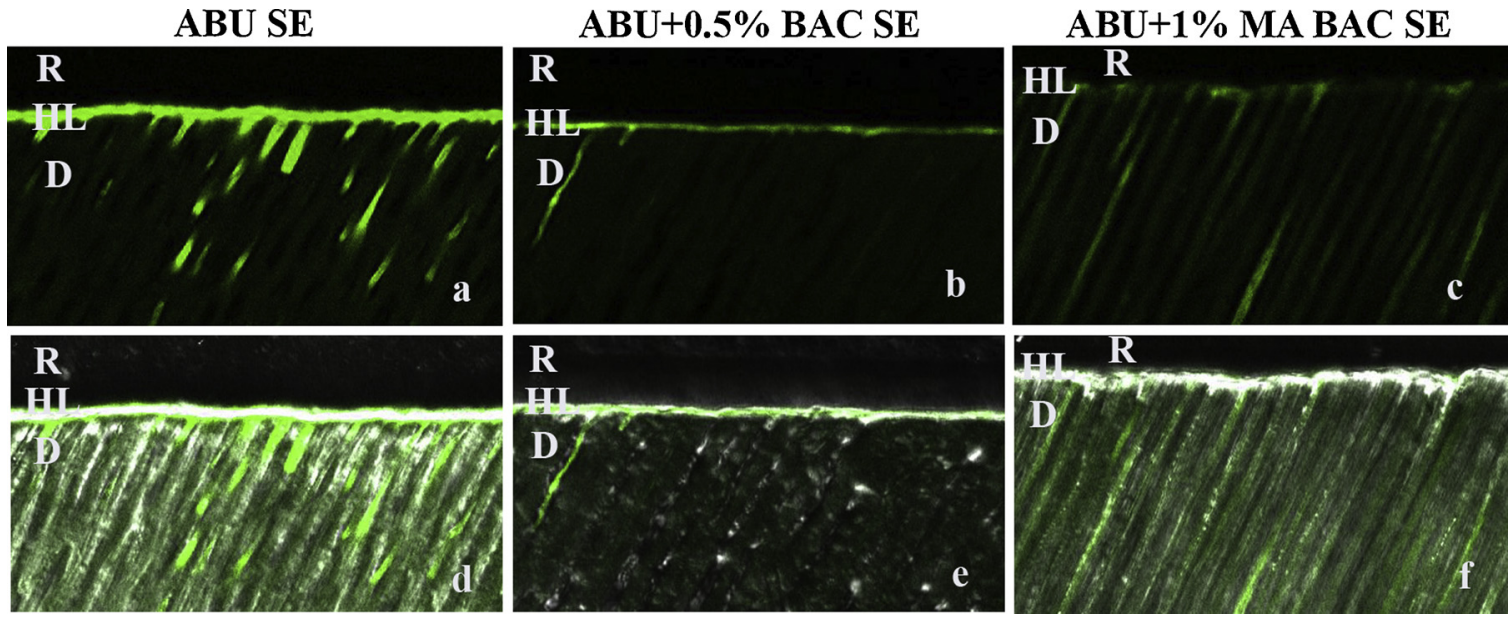

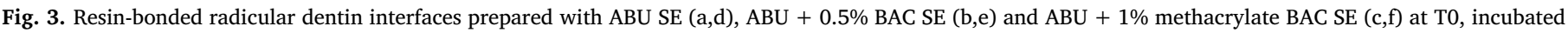

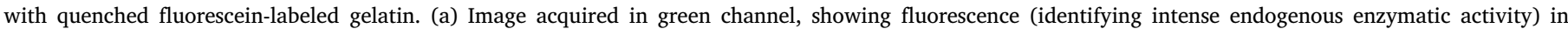

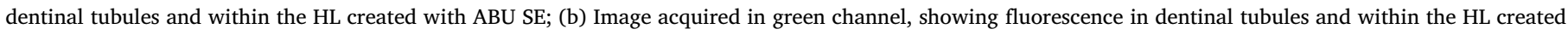

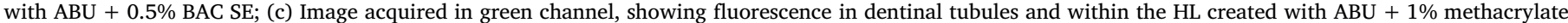

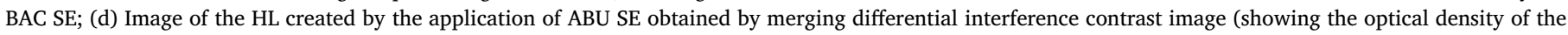

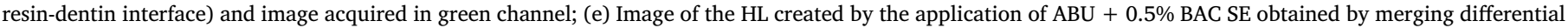

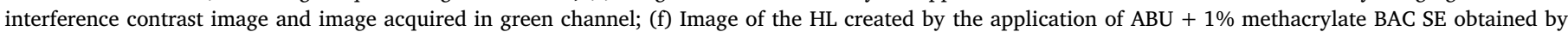

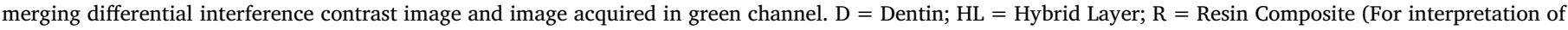
the references to colour in this figure legend, the reader is referred to the web version of this article). 

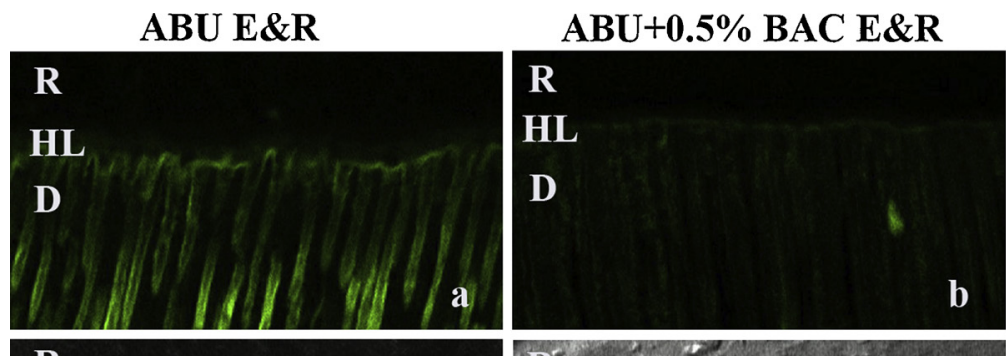

\section{ABU+1\% MA BAC E\&R}
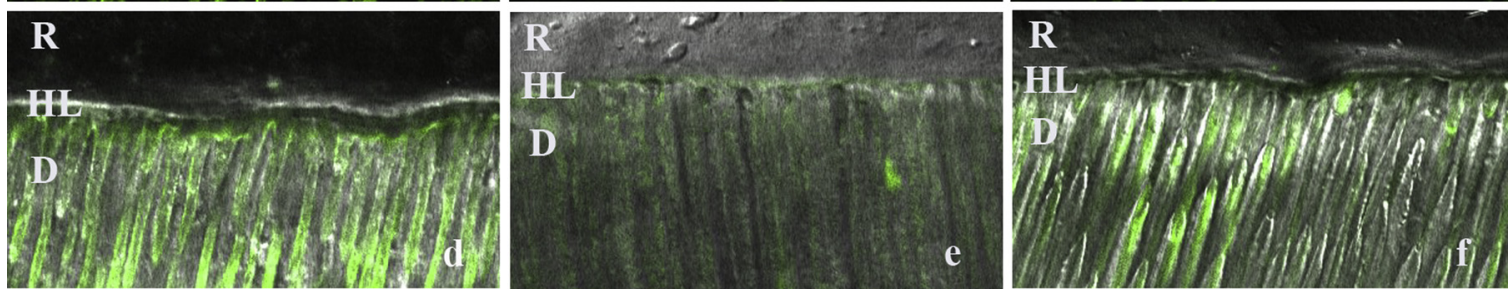

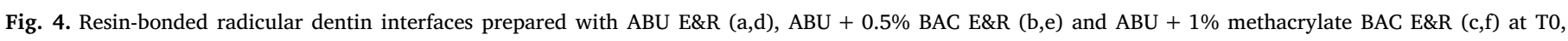

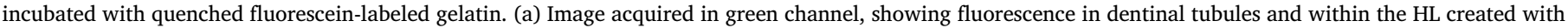

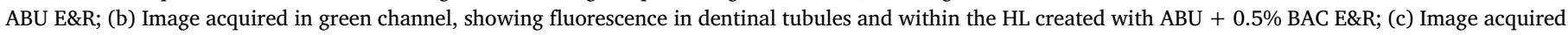

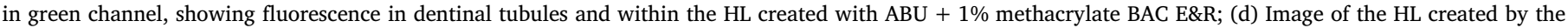

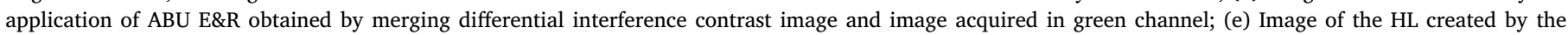

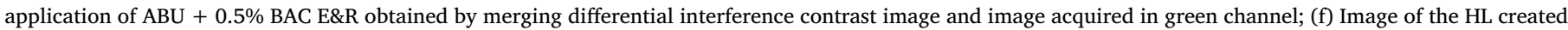

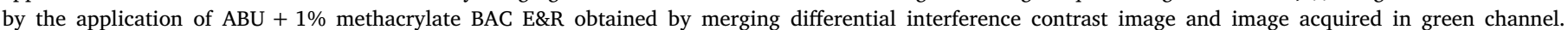

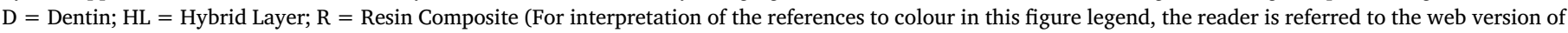
this article).

even in the absence of bacteria and may be attenuated by proteolytic enzyme inhibitors [9].

Benzalkonium chloride has been reported to inhibit MMP activities within the hybrid layer, when applied on acid-etched dentin [27-29]. The quaternary ammonium salt yielded mixed results when it is incorporated into different components of the adhesive system. A commercially available etchant containing 1\% BAC (Bisco) was initially produced by capitalizing on the antimicrobial properties of BAC. Although this antimicrobial etchant was shown to possess anti-collagenolytic potential, dentin bonded with the use of the BAC-containing etchant showed deterioration of the HLs after 6 months [22,30], 12 months [27-29] and 18 months of aging [31]. This may be attributed to the unstable electrostatic interaction between BAC and demineralized dentin and that close to $50 \%$ of the BAC is washed off along with the etchant [18]. Because BAC can be dissolved in ethanol and acetone, and its activity is rather consistent in different $\mathrm{pH}$, it was anticipated that BAC may be incorporated into a large variety of adhesive blends without forfeiting its anti-enzymatic properties [29].

Non-covalently bound molecules, such as non-polymerizable MMP inhibitors, may leach out of the HL. Instability of these non-polymerizable MMP inhibitors compromises long-term anti-proteolytic benefits and results only in delaying, and not preventing the degradation of adhesive interfaces [32,33]. Other strategies have been proposed to circumvent this deleterious issue [28,34,35]. For example, incorporation of compounds that copolymerize with resin monomers in the adhesive blends is promising because the agent becomes
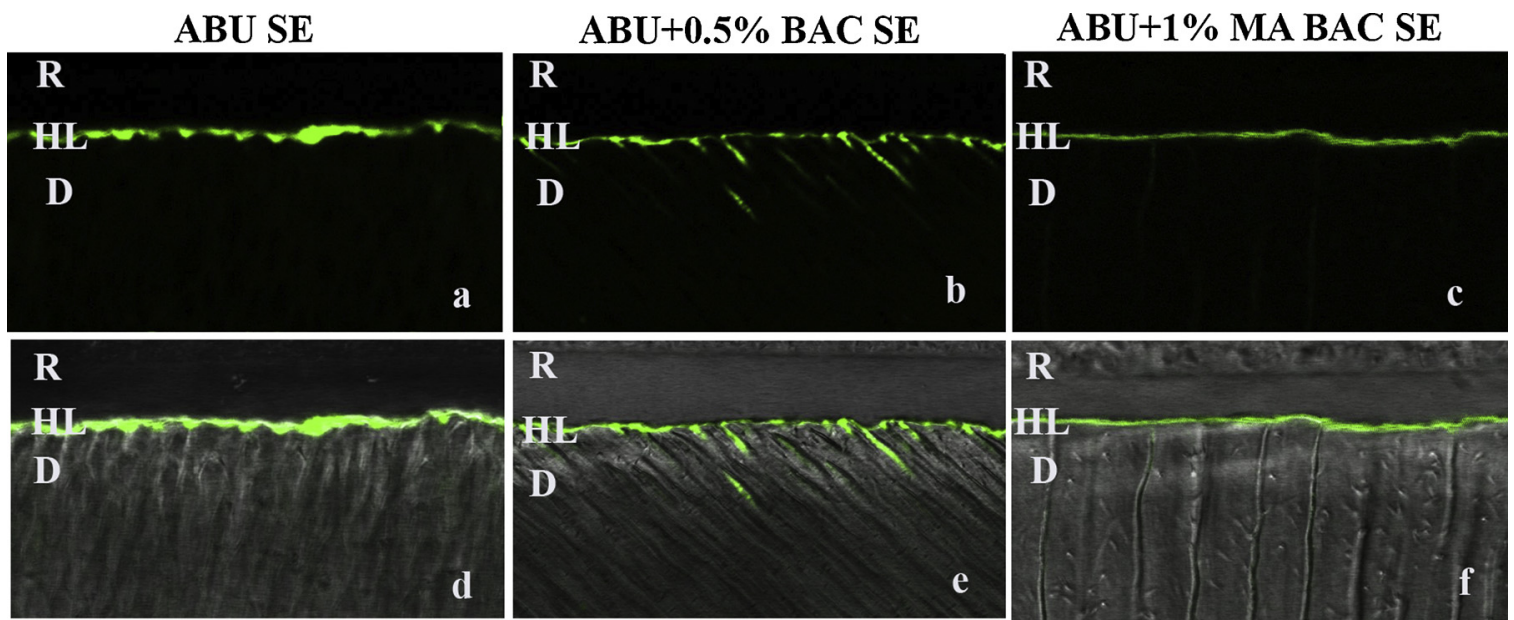

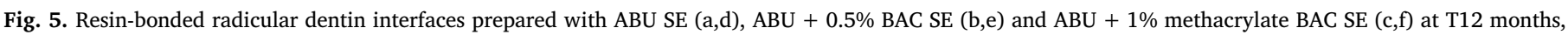

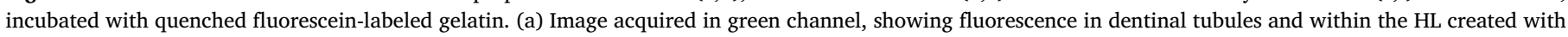

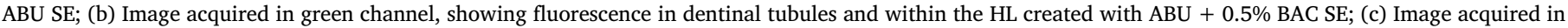

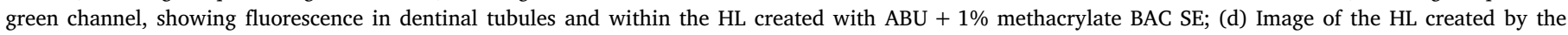

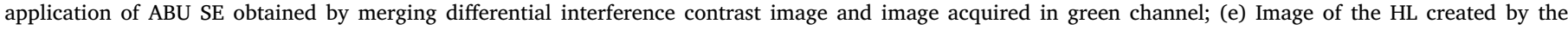

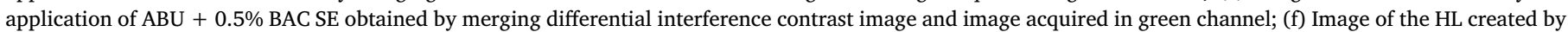

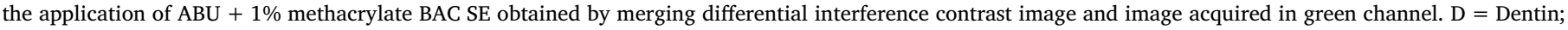

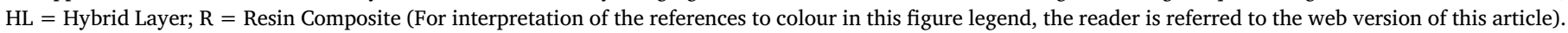



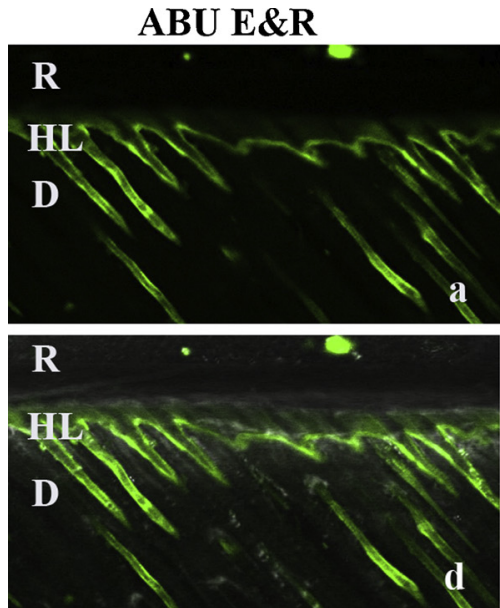

$\mathrm{ABU}+0.5 \%$ BAC E\&R
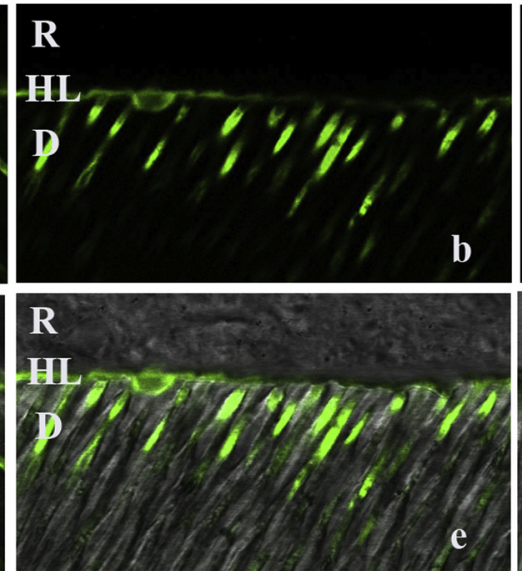

ABU+1\% MA BAC E\&R
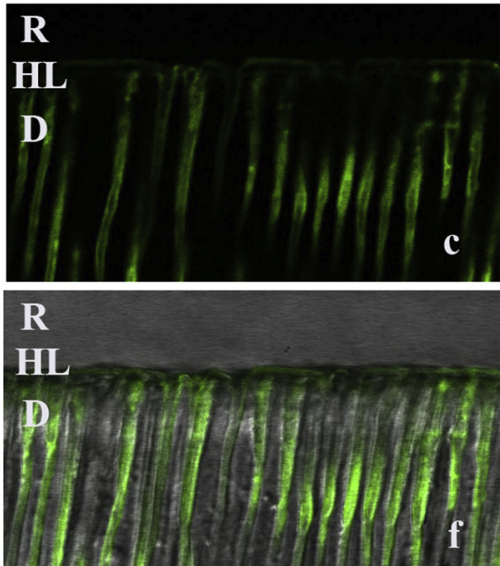

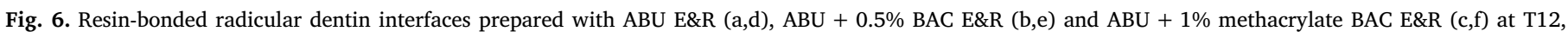

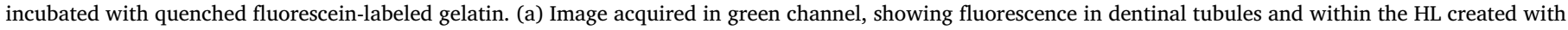

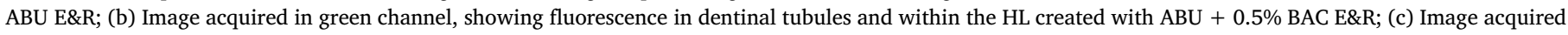

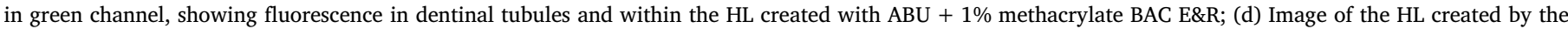

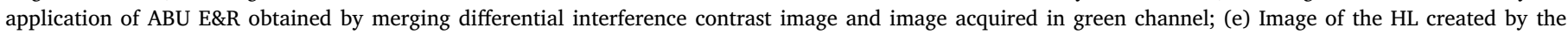

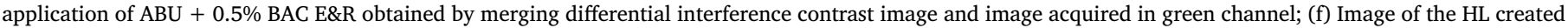

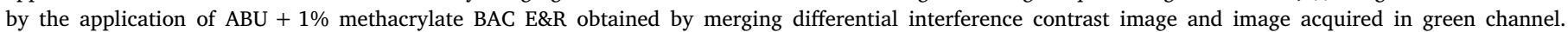

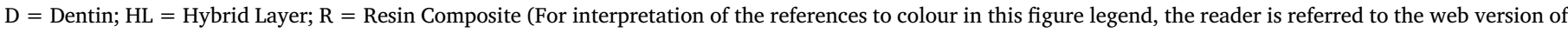
this article).

immobilized within the polymer matrix. In the present study, BAC was blended with a commercially available multi-mode adhesive in different concentrations and formulations $(0.5 \%$ BAC or $1 \%$ BAC methacrylate).

The results of both zymographic analyses demonstrated stronger inhibition of enzymatic activities in the BAC-containing adhesives, when compared with the BAC-free control adhesive. Protease inhibition was the most efficient in experimental groups containing $1 \%$ BAC methacrylate, at the baseline, as well as over time. Several other studies showed the potential of BAC to inhibit endogenous dentinal enzymes $[18,28,31]$ or to prevent collagen degradation [18,22,29]. However, they are not comparable to the present study because of the adoption of different methodologies, and aqueous BAC solution was used in those studies. The present study was the first to examine the effect of incorporation of BAC in an adhesive system on endogenous enzymatic activity. Investigations of bond strength and material properties are required before such an experimental adhesive may be recommended for clinical use.

Incorporation of $0.24-2 \mathrm{wt} \%$ BAC into two different E\&R adhesives (Optibond Solo Plus, Kerr Corp. and All-Bond 3, Bisco) resulted in preservation of their bond strengths to dentin after 18 months of aging. Moreover, in adhesive formulations blended with lower BAC concentrations, increase in bond strength was noted [31]. Adper Single Bond blended with $0.5 \%$ and $1 \%$ of BAC showed no significant decline in bond strength after 6 months [22]. Likewise, Adper Single Bond blended with $0.5 \%$ BAC showed no significant decline in bond strength after 12 months of aging [29]. When a certain concentration of a new component is incorporated into an adhesive, especially in a form of a liquid, there is a risk of jeopardizing the balance between the components of the system. This may have a detrimental effect on the degree of conversion, elastic modulus, tensile stresses and other mechanical properties [36,37]. It is possible that the higher concentration of BAC utilized in the present study adversely affected the polymerization quality and mechanical properties of the resin-dentin interface, both in the E\&R and SE mode. The 1\% methacrylate BAC showed lower bond strength even at baseline compared to the other experimental groups. Contrary to the results of the present study, Sabatini et al. investigated the use of $\mathrm{ABU}$ adhesive system blended with $0.5-2 \%$ BAC or methacrylate BAC in the E\&R mode [28], The authors reported that bond strength was preserved in all the experimental groups after 6 and 12

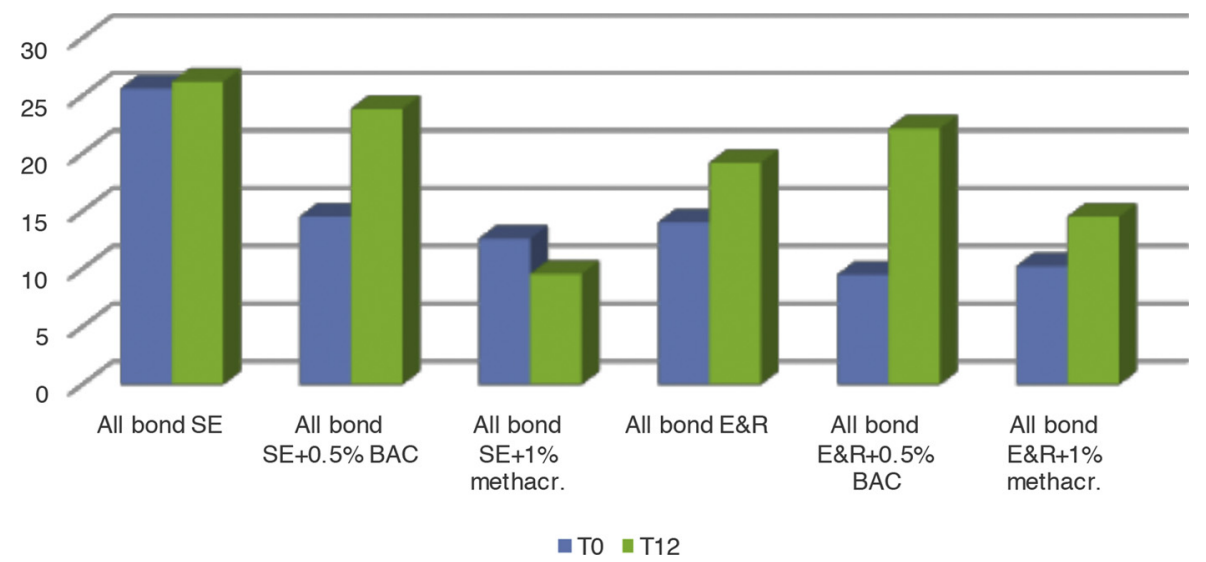

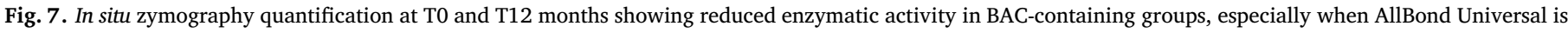
associated with $1 \%$ methacrylate BAC. However, the activity increased with storage in artificial saliva for 12 months. 
Table 1

Microtensile bond strengths of different groups immediately after bonding (T0) and after 12 months of aging (T12) in artificial saliva.

\begin{tabular}{|c|c|c|c|c|c|c|}
\hline \multirow{2}{*}{$\begin{array}{l}\text { Mode } \\
\text { Adhesive }\end{array}$} & \multicolumn{3}{|l|}{$\mathrm{SE}$} & \multicolumn{3}{|l|}{ ER } \\
\hline & $\mathrm{ABU}$ & $\mathrm{ABU}+0.5 \% \mathrm{BAC}$ & $\mathrm{ABU}+1 \% \mathrm{BAC}$ & $\mathrm{ABU}$ & $\mathrm{ABU}+0.5 \% \mathrm{BAC}$ & $\mathrm{ABU}+1 \% \mathrm{BAC}$ \\
\hline T0 & $42.2^{\mathrm{aA}} \pm 16.8$ & $40.5^{\mathrm{aA}} \pm 13.1$ & $40.8^{\mathrm{aA}} \pm 12.4$ & $44.1^{\mathrm{aA}} \pm 13.9$ & $39.7^{\mathrm{aA}} \pm 9.4$ & $36.2^{\mathrm{bA}} \pm 8.7$ \\
\hline T12 & $35.8^{\mathrm{aB}} \pm 17.5$ & $30.2^{\mathrm{aB}} \pm 12.5$ & $21.8^{\mathrm{bB}} \pm 11.6$ & $31.6^{\mathrm{aB}} \pm 14.8$ & $29.0^{\mathrm{aB}} \pm 7.8$ & $17.4^{\mathrm{bB}} \pm 10.5$ \\
\hline
\end{tabular}

Data are presented as means \pm standard deviations, in MPa. Different lower-case letters indicate significant differences $(\mathrm{p}<0.05)$ within the same row, different upper-case letters indicate significant differences $(\mathrm{p}<0.05)$ within the same column.

months of aging. A possible reason for these discrepancies may be due to the manner in which BAC was incorporated into the adhesive, or the difference in light-curing intensities utilized by the two studies.

Incorporation of methacrylate $\mathrm{BAC}$ into $\mathrm{ABU}$ in the present study yielded the worst results in terms of bond strength after aging. However, the same experimental group demonstrated the lowest level of enzymatic activity compared to other groups, especially after aging for 12 months. One can only rationalize that the abhorrent bond strength results are attributed to the poor degree of conversion of BAC methacrylate in the presence of other methacrylate resin monomers in the ABU adhesive. This could have resulted in the leaching of unpolymerized BAC methacrylate resin monomer from the resin-dentin interface. Apart from potentially killing cells in the vital dental pulp, the resin-dentin interface becomes porous after leaching of the unpolymerized resin monomer. This, in turn, facilitates ingress of water and salivary enzymes into the HL and subsequent hydrolysis of the other polymerized resin components. Although leaching of the BAC methacrylate monomer could have resulted in better MMP inhibition, the overall result showed no contribution to the longevity of resindentin bonds.

\section{Conclusions}

Although the BAC-containing adhesive formulations showed inhibitory potential on dentinal endogenous enzymes at the baseline, this property did not seem to be long-lasting. The MMP inhibition potential of the experimental adhesive formulation was not reflected by the bond strength results. Overall, the experiment shows that adhesives blended with MMP inhibitors should be extensively tested before translation to clinical use.

\section{Conflict of interest statement}

We wish to confirm that there are no known conflicts of interest associated with this publication and there has been no significant financial support for this work that could have influenced its outcome.

\section{References}

[1] M.G. Rasines Alcaraz, A. Veitz-Keenan, P. Sahrmann, P.R. Schmidlin, D. Davis, Z. Iheozor-Ejiofor, Direct composite resin fillings versus amalgam fillings for permanent or adult posterior teeth, Cochrane Database Syst. Rev. 31 (2014) CD005620, , https://doi.org/10.1002/14651858.CD005620.pub2.

[2] T. Maravic, A. Mazzoni, A. Comba, N. Scotti, V. Checchi, L. Breschi, How stable is dentin as a substrate for bonding? Curr. Oral Heal. Rep. 4 (2017) 248-257, https:// doi.org/10.1007/s40496-017-0149-8.

[3] Y. Liu, L. Tjäderhane, L. Breschi, A. Mazzoni, N. Li, J. Mao, D.H. Pashley, F.R. Tay, Limitations in bonding to dentin and experimental strategies to prevent bond degradation, J. Dent. Res. 90 (2011) 953-968, https://doi.org/10.1177/ 0022034510391799.

[4] L. Breschi, T. Maravic, S.R. Cunha, A. Comba, M. Cadenaro, L. Tjäderhane, D.H. Pashley, F.R. Tay, A. Mazzoni, Dentin bonding systems: from dentin collagen structure to bond preservation and clinical applications, Dent. Mater. 34 (2018) 78-96, https://doi.org/10.1016/j.dental.2017.11.005.

[5] N. Nakabayashi, K. Kojima, E. Masuhara, The promotion of adhesion by the in filtration of monomers into tooth substrates, J. Biomed. Mater. Res. 16 (1982) 265-273.

[6] S.R. Armstrong, M.A. Vargas, I. Chung, D.H. Pashley, J.A. Campbell, J.E. Laffoon, F. Qian, Resin-dentin interfacial ultrastructure and microtensile dentin bond strength after five-year water storage, Oper. Dent. 29 (2004) 705-712.

[7] M. Hashimoto, H. Ohno, M. Kaga, K. Endo, H. Sano, H. Oguchi, In vivo degradation of resin-dentin bonds in humans over 1 to 3 years, J. Dent. Res. 79 (2000) 1385-1391, https://doi.org/10.1177/00220345000790060601.

[8] J. De Munck, K. Van Landuyt, M. Peumans, A. Poitevin, P. Lambrechts, M. Braem, B. Van Meerbeek, A critical review of the durability of adhesion to tooth tissue: methods and results, J. Dent. Res. 84 (2005) 118-132, https://doi.org/10.1177/ 154405910508400204

[9] D. Pashley, F. Tay, C. Yiu, M. Hashimoto, L. Breschi, R. Carvalho, Collagen degradation by host-derived enzymes during aging, J. Dent. Res. 83 (2004) 216-221.

[10] A. Mazzoni, V. Angeloni, F.M. Apolonio, N. Scotti, L. Tjäderhane, A. TezvergilMutluay, R. Di Lenarda, F.R. Tay, D.H. Pashley, L. Breschi, Effect of carbodiimide (EDC) on the bond stability of etch-and-rinse adhesive systems, Dent. Mater. 29 (2013) 1040-1047, https://doi.org/10.1016/j.dental.2013.07.010.

[11] L. Tjäderhane, F.D. Nascimento, L. Breschi, A. Mazzoni, I.L.S. Tersariol, S. Geraldeli, A. Tezvergil-Mutluay, M.R. Carrilho, R.M. Carvalho, F.R. Tay, D.H. Pashley, Optimizing dentin bond durability: control of collagen degradation by matrix metalloproteinases and cysteine cathepsins, Dent. Mater. 29 (2013) 116-135, https:// doi.org/10.1016/j.freeradbiomed.2008.10.025.The.

[12] M.R.O. Carrilho, S. Geraldeli, F. Tay, M. de Goes, R. Carvalho, L. Tjäderhane, In vivo preservation of hybrid layer by chlorhexidine, J. Dent. Res. 86 (2007) 529-533.

[13] Y. Nishitani, M. Yoshiyama, B. Wadgaonkar, L. Breschi, F. Mannello, A. Mazzoni, R. Carvalho, L. Tjäderhane, F. Tay, D. Pashley, Activation of gelatinolytic/collagenolytic activity in dentin by self etching adhesives, Eur. J. Oral Sci. 114 (2006) $160-166$

[14] L. Breschi, F. Cammelli, E. Visintini, A. Mazzoni, M. Carrilho, M. Cadenaro, S. Foulger, F.R. Tay, Influence of chlorhexidine concentration on the durability of etch-and-rinse dentin bonds: a 12-month in vitro study, J. Adhes. Dent. 11 (2009) 191-198.

[15] L. Breschi, P. Martin, A. Mazzoni, F. Nato, M. Carrilho, L. Tjäderhane, E. Visintini, M. Cadenaro, F.R. Tay, E.D.S. Dorigo, D.H. Pashley, Use of a specific MMP-inhibitor (galardin) for preservation of hybrid layer, Dent. Mater. 26 (2010) 571-578, https://doi.org/10.1016/j.dental.2010.02.007.

[16] A. Mazzoni, V. Angeloni, N. Sartori, S. Duarte, T. Maravic, L. Tjäderhane, D.H. Pashley, F.R. Tay, L. Breschi, Substantivity of carbodiimide inhibition on dentinal enzyme activity over time, J. Dent. Res. 96 (2017) 902-908, https://doi. org/10.1177/0022034517708312.

[17] A. Cova, L. Breschi, F. Nato, A. Ruggeri, M. Carrilho, L. Tjaderhane, C. Prati, R. Di Lenarda, F. Tay, D. Pashley, A. Mazzoni, Effect of UVA-activated riboflavin on dentin bonding, J. Dent. Res. 90 (2011) 1439-1445, https://doi.org/10.1177/ 0022034511423397.

[18] A. Tezvergil-Mutluay, M.M. Mutluay, L.S. Gu, K. Zhang, K.A. Agee, R.M. Carvalho, A. Manso, M. Carrilho, F.R. Tay, L. Breschi, B.I. Suh, D.H. Pashley, The anti-MMP activity of benzalkonium chloride, J. Dent. 39 (2011) 57-64, https://doi.org/10. 1016/j.jdent.2010.10.003.

[19] A. Tezvergil-Mutluay, M.M. Mutluay, K.A. Agee, R. Seseogullari-Dirihan, T. Hoshika, M. Cadenaro, L. Breschi, P. Vallittu, F.R. Tay, D.H. Pashley, Carbodiimide cross-linking inactivates soluble and matrix-bound MMPs, in vitro, J. Dent. Res. 91 (2012) 192-196, https://doi.org/10.1177/0022034511427705.

[20] L. Breschi, Chlorhexidine application to stabilize the adhesive interface: why and how? J. Adhes. Dent. 15 (2013) 492, https://doi.org/10.3290/j.jad.a32067.

[21] J. Kanca III, One Step bond strength to enamel and dentin, Am. J. Dent. 10 (1997).

[22] C. Sabatini, J. Kim, P.O. Alias, In vitro evaluation of benzalkonium chloride in the preservation of adhesive interfaces, Oper. Dent. 39 (2014) 283-290, https://doi. org/10.2341/13-131-LR.

[23] A. Mazzoni, P. Scaffa, M. Carrilho, L. Tjäderhane, R. Di Lenarda, A. Polimeni, A. Tezvergil-Mutluay, F.R. Tay, D.H. Pashley, L. Breschi, Effects of etch-and-rinse and self-etch adhesives on dentin MMP-2 and MMP-9, J. Dent. Res. 92 (2013) 82-86, https://doi.org/10.1177/0022034512467034.

[24] A. Mazzoni, F.M. Apolonio, V.P.A. Saboia, S. Santi, V. Angeloni, V. Checchi, R. Curci, R. Di Lenarda, F.R. Tay, D.H. Pashley, L. Breschi, Carbodiimide inactivation of MMPs and effect on dentin bonding, J. Dent. Res. 93 (2014) 263-268, https://doi.org/10.1177/0022034513516465.

[25] A. Mazzoni, F. Nascimento, M. Carrilho, I. Tersariol, V. Papa, L. Tjaderhane, R. Di Lenarda, F. Tay, D. Pashley, L. Breschi, MMP activity in the hybrid layer detected with in situ zymography, J. Dent. Res. 91 (2012) 467-472, https://doi.org/10. 1177/0022034512439210.

[26] M.R.O. Carrilho, R.M. Carvalho, M.F. de Goes, V. di Hipólito, S. Geraldeli, F.R. Tay, D.H. Pashley, L. Tjäderhane, Chlorhexidine preserves dentin bond in vitro, J. Dent. Res. 86 (2007) 90-94, https://doi.org/10.1177/154405910708600115. 
[27] N. Tekçe, S. Tuncer, M. Demirci, S. Balci, Do matrix metalloproteinase inhibitors improve the bond durability of universal dental adhesives? Scanning 38 (2016) 535-544, https://doi.org/10.1002/sca.21293.

[28] C. Sabatini, D.H. Pashley, Aging of adhesive interfaces treated with benzalkonium chloride and benzalkonium methacrylate, Eur. J. Oral Sci. 123 (2015) 102-107, https://doi.org/10.1111/eos.12168.

[29] C. Sabatini, P.A. Ortiz, D.H. Pashley, Preservation of resin-dentin interfaces treated with benzalkonium chloride adhesive blends, Eur. J. Oral Sci. 123 (2015) 108-115, https://doi.org/10.1111/eos.12176.

[30] M. El Gezawi, R. Haridy, E. Abo Elazm, F. Al-Harbi, M. Zouch, D. Kaisarly, Microtensile bond strength, 4-point bending and nanoleakage of resin-dentin interfaces: effects of two matrix metalloproteinase inhibitors, J. Mech. Behav. Biomed. Mater. 78 (2018) 206-213, https://doi.org/10.1016/J.JMBBM.2017.11. 024.

[31] C. Sabatini, S.K. Patel, Matrix metalloproteinase inhibitory properties of benzalkonium chloride stabilizes adhesive interfaces, Eur. J. Oral Sci. 121 (2013) 610-616, https://doi.org/10.1111/eos.12089.

[32] H. Sano, T. Takatsu, B. Ciucchi, J. Honer, W. Matthews, D. Pashley, Nanoleakage: leakage within the hybrid layer, Oper. Dent. 20 (1995) 18-25.

[33] F.R. Tay, N.M. King, K. Chan, D.H. Pashley, How can nanoleakage occur in selfetching adhesive systems that demineralize and infiltrate simultaneously? J. Adhes. Dent. 4 (2002) 255-269.

[34] D. Pashley, F. Tay, S. Imazato, How to increase the durability of resin-dentin bonds, Compend. Contin. Educ. Dent. 32 (2011) 60-64.

[35] J.M. Antonucci, D.N. Zeiger, K. Tang, S. Lin-Gibson, B.O. Fowler, N.J. Lin, Synthesis and characterization of dimethacrylates containing quaternary ammonium functionalities for dental applications, Dent. Mater. 28 (2012) 219-228, https://doi. org/10.1016/j.dental.2011.10.004.

[36] M. Cadenaro, D.H. Pashley, G. Marchesi, M. Carrilho, F. Antoniolli, A. Mazzoni, F.R. Tay, R. Di Lenarda, L. Breschi, Influence of chlorhexidine on the degree of conversion and E-modulus of experimental adhesive blends, Dent. Mater. 25 (2009) 1269-1274, https://doi.org/10.1016/j.dental.2009.05.008.

[37] M. Cadenaro, T. Maravic, A. Comba, A. Mazzoni, L. Fanfoni, T. Hilton, J. Ferracane, L. Breschi, The role of polymerization in adhesive dentistry, Dent. Mater. 35 (2018) e1-e22, https://doi.org/10.1016/j.dental.2018.11.012. 